\title{
Anti-ship Missile Target Dynamic RCS Statistical Distribution Fitting Technique
}

\author{
Wang Hao1, Shao Jixing1 \& Hu Yue1 \\ 1PLA, No. 92941 Troop, Liaoning Hu Ludao 125001, China.
}

Keywords:radar cross section (RCS), statistical model, fitting technique, test hypothesis.

\begin{abstract}
To deal with the construction of the anti-ship missile target dynamic RCS statistical model, target RCS fitting and construction method was given by introducing the hypothesis test. Kolmogorov-Smirnov test method was given to resolve the distribution fitting test problem. Finally, the method was verified by simulation.
\end{abstract}

\section{Introduction}

Radar echo is always related with target radar RCS. RCS is related with the target motion regular and work frequency[1-3]. Thus, the statistical model can not be described fixed. However, the rule can be analysed and grasped[4]. Firstly, the characteristic parameters, probability density curve and cumulated density curve were given. Secondly, RCS statistical distribution method was constructed. Finally, the method was verified by simulation and the models were compared by the fitting result to build a more accurate statistical model.

\section{RCS statistical parameters[5]}

The discussed model is given as follows: Some statistical characteristic are used to describe RCS measurement. Common statistical parameters include mean value $\bar{\sigma}$, standard error $\sigma_{\text {std }}$, minimum value $\sigma_{\min }$, maximum value $\sigma_{\max }$, probability density function(PDF), cumulated distribution function and so on.

$$
\begin{gathered}
\bar{\sigma}=\frac{1}{N} \sum_{i=1}^{N} \sigma_{i} \\
\sigma_{\text {std }}=\left[\frac{\frac{1}{N}\left(\sigma_{i}-\bar{\sigma}\right)^{2}}{N-1}\right]^{1 / 2} \\
\sigma_{\text {min }}=\min \left(\sigma_{1}, \sigma_{2}, \ldots, \sigma_{n}\right) \\
\sigma_{\text {max }}=\max \left(\sigma_{1}, \sigma_{2}, \ldots, \sigma_{n}\right)
\end{gathered}
$$

Probability density function (PDF) and cumulated distribution function(CDF) when defined in the internal of $\sigma_{0}$ and $\sigma_{0}+\mathrm{d} \sigma$ are given as follows,

$$
\begin{gathered}
P\left(\sigma_{0} \leq \sigma \leq \sigma_{0}+\mathrm{d} \sigma\right)=\int_{\sigma_{0}}^{\sigma_{0}+\mathrm{d} \sigma} \operatorname{PDF}(\sigma) \mathrm{d} \sigma \\
\operatorname{CDF}(\sigma)=\int_{-\infty}^{\sigma} \operatorname{PDF}(\sigma) \mathrm{d} \sigma
\end{gathered}
$$

\section{Measurement fluctuation model[6]}

Since target radar RCS is sensitive with the variety of the target pose, target radar RCS is a fluctuation variable. To analyze and evaluate the influence of the target RCS fluctuation on the radar detection performance, some statistical model to describe target RCS fluctuation is adopted commonly. RCS fluctuation model can be described as two developing eras. Five classical models such as non fluctuation model and Swerling $\mathrm{I} \sim \mathrm{IV}$ are constructed in the first era. The second era 
models are $\chi^{2}$ (Chi2square) distribution model, logarithm normal school (Log2normal) model, Rayleigh distribution model and so on.

$\chi^{2}$ distribution density function is

$$
P(\sigma)=\frac{1}{\Gamma(k)} \frac{k}{\bar{\sigma}}\left[\frac{k \sigma}{\bar{\sigma}}\right]^{k-1} \exp \left\{-\frac{k \sigma}{\bar{\sigma}}\right\}, \sigma>0
$$

where, $\bar{\sigma}$ is the mean value of $\sigma$, $\mathrm{k}$ is double free degree (higher the value is, more tempered the fluctuation is ), $2 \mathrm{k}$ is the free degree of the $\chi^{2}$ distribution.

The Logarithm normal probability density function is given by

$$
P(\sigma)=\frac{1}{\sqrt{2 \pi} s_{d} \sigma} \exp \left\{-\frac{1}{2 s_{d}^{2}}\left[\operatorname{In}\left(\frac{\sigma}{\sigma_{m}}\right)\right]^{2}\right\}, \sigma>0
$$

where, $\sigma_{m}$ is media value of $\sigma, s_{d}$ is the standard error of $\operatorname{In}\left(\frac{\sigma}{\sigma_{m}}\right)$.

The Rayleigh probability density function is given by

$$
f(x)=\left\{\begin{array}{cc}
\frac{x}{\sigma^{2}} \exp \left(-\frac{x^{2}}{2 \sigma^{2}}\right) & x \geq 0 \\
0 & x<0
\end{array}\right.
$$

where $\sigma^{2}$ is known as the fading envelope of the Rayleigh distribution.

The Weibull probability density function is given by

$$
f(x \mid a, b)=b a^{-b} x^{b-1} e^{-\left(\frac{x}{a}\right)^{b}} I(0, \infty)(x)
$$

where $\mathrm{a}$ is a scale parameter, $\mathrm{b}$ is a shape parameter.

\section{Target RCS fitting and construction method}

For a specific radar target, its RCS value is changing with the pose variety. Based on the data process, variable distribution test is given. Since the RCS distribution function is unknown, the hypothesis test is constructed based on the certain significance degree.

$$
\begin{aligned}
& \mathrm{H}_{0}: F(x)=F_{0}(x) \\
& \mathrm{H}_{1}: F(x) \neq F_{0}(x)
\end{aligned}
$$

where, $\mathrm{H}_{0}$ is the null hypothesis, $\mathrm{H}_{1}$ is the alternative hypothesis, $F_{0}(x)$ is a given distribution model.

Based on the hypothesis test, through the process, the probability distribution curve can be given by the measurements. According to the given distribution model, the parameters of the distribution curve can be fitted by a certain fitting algorithm. Kolmogorov-Smirnov test method[7] are given to resolve the distribution fitting test problem. Matlab calls these two test methods as KolmogorovSmirnov test and provides two test functions like kstest and kstest2.

The fitting steps are as follows,

Step1: Choose the distribution model,

Step2: Confirm the hypothesis test,

Step3: Probability distribution curve is obtained by measurement statistical model,

Step4: Distance value is computed between the fitting curve and the chosen distribution,

Step5:Compare the distance value and determining threshold to judge whether to obey the given distribution,

Step6:Parameters of the given distribution can be obtained by the fitting algorithm.

\section{Simulation results and analysis}

The simulation results is given based on the measurement used in ref.[8]. Only the influence of the azimuth is considered here. The distribution models used here included logarithm normal school, 
Rayleigh distribution and Weibull distribution. Fitting methods adopted here is Maximum likelihood estimation. RCS cumulated function fitted in linearity space are given in Fig1, Fig.2 and Fig.3 respectively. RCS cumulated function fitted in logarithm space are given in Fig.4, Fig.5 and Fig. 6 respectively. Fitting test results for each distribution model in linearity space are shown in table 1 and the results in logarithm space are shown in table 2.

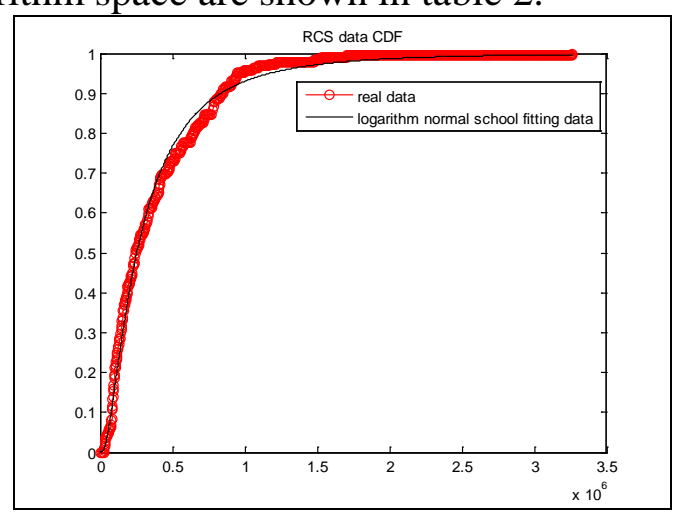

Fig. 1 RCS cumulated function Fitted based on logarithm normal school in linearity space $\left(\mathrm{m}^{2}\right)$

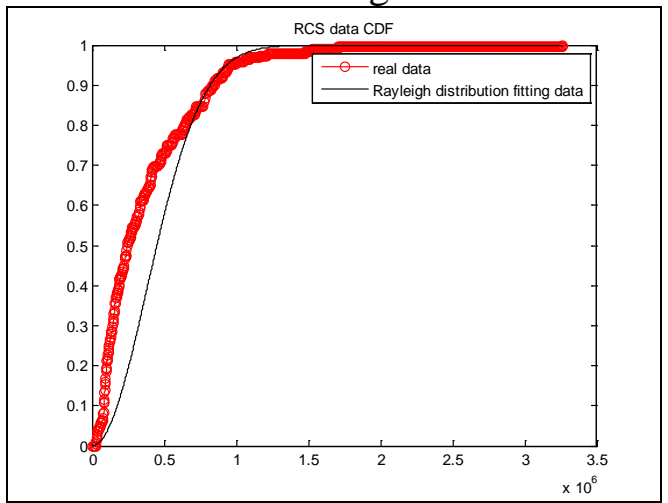

Fig. 2 RCS cumulated density function Fitted based on Rayleigh distribution in linearity space $\left(\mathrm{m}^{2}\right)$

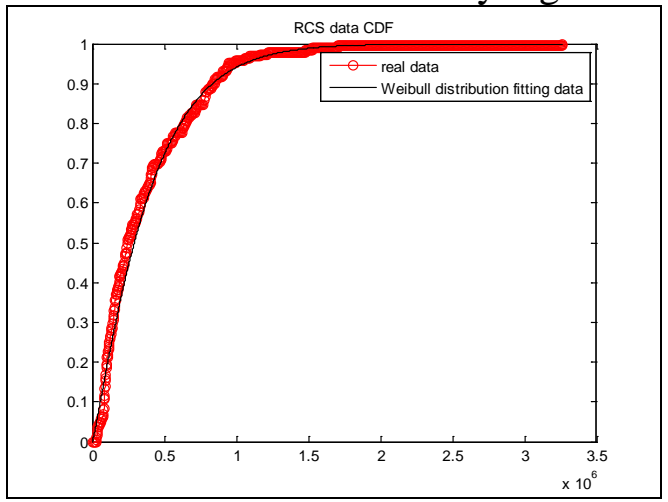

Fig. 3 RCS cumulated density function Fitted based on weibull distribution in linearity space

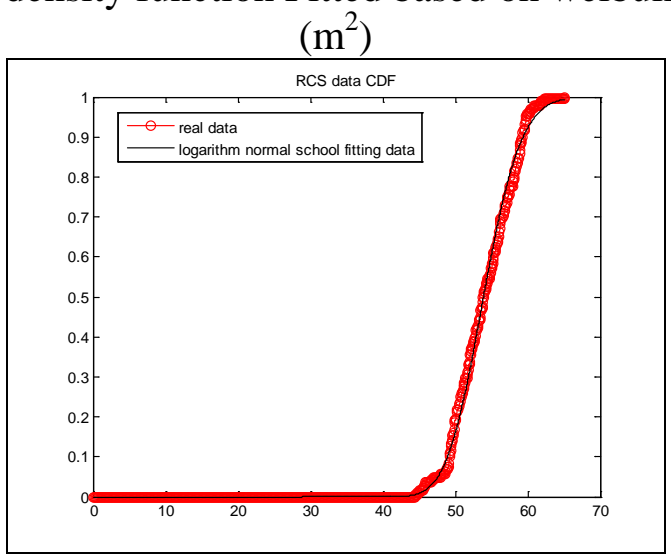

Fig. 4 RCS cumulated function Fitted based on logarithm normal school in logarithm space $\left(\mathrm{dBm}^{2}\right)$ 


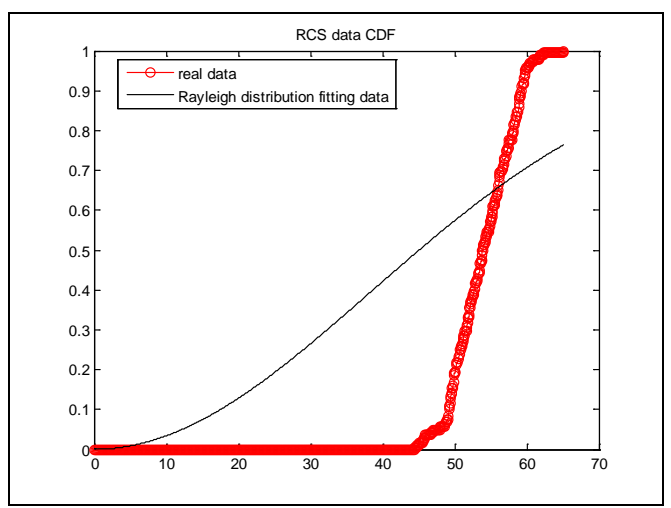

Fig. 5 RCS cumulated density function Fitted based on Rayleigh distribution in logarithm space $\left(\mathrm{dBm}^{2}\right)$

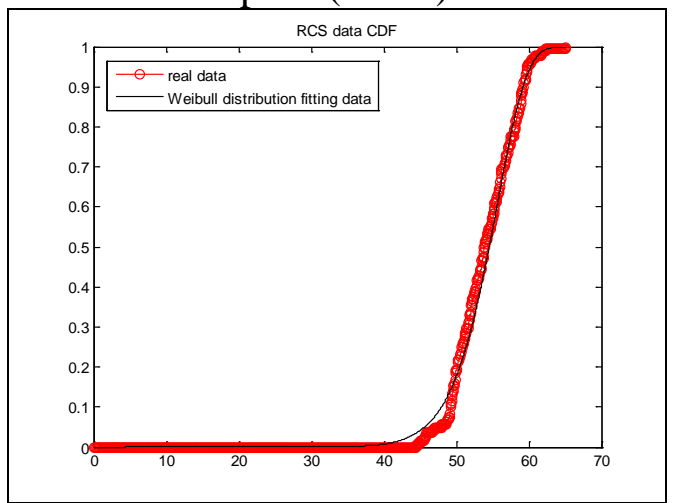

Fig. 6 RCS cumulated density function Fitted based on weibull distribution in logarithm space $\left(\mathrm{dBm}^{2}\right)$

Table 1: Fitting test results for each distribution model in linearity space

\begin{tabular}{ccccc}
\hline $\begin{array}{c}\text { Distribution } \\
\text { model }\end{array}$ & $\begin{array}{c}\text { Distance } \\
\text { statistics }\end{array}$ & $\begin{array}{c}\text { Determining } \\
\text { threshold }\end{array}$ & $\begin{array}{c}\text { Comparison } \\
\text { result }\end{array}$ & Parameters \\
\hline $\begin{array}{c}\text { Rayleigh } \\
\text { Logarithm } \\
\text { normal }\end{array}$ & 0.31655 & 0.097862 & $>$ & -- \\
weibull & 0.05745 & 0.097862 & $<$ & $12.4454,0.930$ \\
& 0.07739 & 0.097862 & $<$ & $\begin{array}{c}\text { 402639.1, } \\
1.13\end{array}$ \\
\hline
\end{tabular}

Table 2: Fitting test results for each distribution model in logarithm space

\begin{tabular}{ccccc}
\hline $\begin{array}{c}\text { Distribution } \\
\text { model }\end{array}$ & $\begin{array}{c}\text { Distance } \\
\text { statistics }\end{array}$ & $\begin{array}{c}\text { Determining Comparison } \\
\text { threshold }\end{array}$ & $\begin{array}{c}\text { Parameters } \\
\text { result }\end{array}$ & \\
\hline Rayleigh & 0.49631 & 0.097862 & $>$ & - \\
$\begin{array}{c}\text { Logarithm } \\
\text { normal }\end{array}$ & 0.05655 & 0.097862 & $<$ & 3.9871, \\
weibull & 0.066037 & 0.097862 & $<$ & $\begin{array}{c}\text { ( } 0.075311 \\
\end{array}$ \\
\hline
\end{tabular}

It can be obtained from the figures and tables,

(1)In both the linearity space and logarithm space, the statistical model can be fitted well by using logarithm normal school and weibull distribution, not the Rayleigh distribution.

(2)In linearity space, the distance statistics between logarithm normal school fitting model and the real measurement is 0.05745 . While, the distance statistics between weibull distribution fitting model and the real measurement is 0.07739. In logarithm space, the distance statistics between logarithm normal school fitting model and the real measurement is 0.05655 . While, the distance statistics between weibull distribution fitting model and the real measurement is 0.066037 . Thus, 
the logarithm normal school is better for model fitting in both linearity space and logarithm space. Thus, the logarithm normal school is better for model fitting.

\section{Conclusions}

Anti-ship missile target RCS statistical characteristic was studied and fitting technique was proposed base on test hypothesis method. Simulation results showed that the proposed method was effective.

\section{References}

[1] Yardim, F.E. \& Akcam, N. Estimation of Radar Cross-Section in Rayleigh, MIE and Optical Regions by the 2-D-FDTD Simulation. Antennas and Propagation, IEEE Transactions on, 62(11), pp.5782 - $5789,2014$.

[2] Cakir, G.; Cakir, M. \& Sevgi, L. Radar Cross Section (RCS) Modeling and Simulation, Part 2: A Novel FDTD-Based RCS Prediction Virtual Tool for the Resonance Regime. Antennas and Propagation Magazine, IEEE, 50(2), pp. 81-94,2008.

[3] E. Knott, M. Tuley, \& J. Shaeffer, Radar Cross Section, 2nd ed, Norwood, MA: Artech House,1993.

[4] Zaman, M.A. \& Matin, M.A. Radar cross section calculation of a shell-shaped projectile using Bézier curves and physical optics. Electrical \& Computer Engineering (ICECE), 7th International Conference on, pp 690-693,2012.

[5] Song Guang, Zhang De-bao \& Li Ming. Analysis of statistical model for RCS of surface ship. Shipboard electronic countermeasure, 34(2), pp.73-74,78,2011.

[6] Zeng Yong-hu, Wang Guo-yu, Chen Yong-guang \& Wang Lian-dong. The analysis of target's RCS fluctuation based on $\chi^{2}$ distribution. Radar science and technology, 5(2), pp.115$117,123,2007$.

[7] Cao Zhan-hui, Li Yong-hua \& Li Yan-jun. Kolmogorov entropy of space object RCS. Computer Engineering and Applications, 44(14), pp.225-227,2008.

[8] Chen Cong \& Gao Huo-tao. Estimation of ship targets radar cross sections for HFSWR. Chinese journal of radio science, 24(4), pp.675-681,2009. 